\title{
Caracterização da empatia em internos de medicina geral e familiar
}

\author{
Nuno Basílio, ${ }^{1}$ Ana Sofia Vitorino, ${ }^{2}$ José Mendes Nunes ${ }^{3-4}$
}

\begin{abstract}
RESUMO
Objetivos: Caracterizar o nível de empatia dos internos do primeiro e último ano da formação específica (FE) em medicina geral e familiar (MGF) e comparação entre os referidos grupos.

Tipo de estudo: Observacional, analítico e transversal.

Local: Administrações Regionais de Saúde (ARS) de Portugal.

População: Internos de MGF em formação específica nas ARS de Portugal.

Métodos: Aplicou-se um questionário anónimo, de autopreenchimento, utilizando a Jefferson Scale of Physician Empathy (JSPE), adaptada e validada para a população médica portuguesa, a uma amostra de conveniência de internos selecionada do universo pretendido. Os inquéritos foram aplicados presencialmente ou via e-mail pessoal. A análise estatística foi feita através de SPSS (R) v. 19.0.

Resultados: Foram recolhidos 304 questionários: 84 do grupo de internos que iniciaram a FE em 2012 (taxa de resposta (TR)=20,6\%) e 220 do grupo de internos que iniciaram a FE em 2016 (TR=42,3\%), pertencendo a maioria dos internos de cada grupo à ARS de Lisboa e Vale do Tejo ( $52,4 \%$ e $61,4 \%$, respetivamente). Entre ambos os grupos não se verificaram diferenças estatisticamente significativas na variável sexo $(p=0,466)$. Os níveis medianos de empatia (ME) obtidos com a aplicação da JSPE não apresentam diferenças estatisticamente significativas nos dois grupos $[\mathrm{ME}(2012)=119,00 ; \mathrm{ME}(2016)=118,00 ; p=0,678]$ e a experiência prévia na área da comunicação não se relaciona com melhor pontuação $(p=0,610)$. Os elementos do sexo feminino apresentaram níveis de empatia estatisticamente superiores $(p=0,008)$.

Conclusões: Os resultados obtidos são comparáveis a estudos nacionais e internacionais com estudantes de medicina. A capacidade empática não parece variar com a formação específica ou com a formação pré-graduada. A baixa taxa de resposta não permitiu atingir representatividade, limitando a robustez dos resultados. O presente estudo é o ponto de partida para a análise da variação da capacidade empática ao longo da formação específica.
\end{abstract}

Palavras-chave: Empatia; Internato; Medicina geral e familiar; Cuidados de saúde primários.

\section{INTRODUÇÃO}

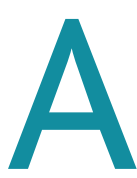

empatia consiste no processo de comunicar a uma pessoa o sentimento de que está a ser compreendida. ${ }^{1}$ É um fator importante no estabelecimento das relações interpessoais, sendo ainda mais determinante na relação médico-doente, ${ }^{2}$ no aumento da satisfação, da capacidade diagnóstica e no empoderamento dos doentes. ${ }^{3}$ Este processo foi descrito por Barrett-Lennard na perspetiva de ciclo empático caracterizado por um contínuo entre três fases: escuta empática, compreensão/expressão empática e consciencialização por parte do paciente deste processo comunicacional com o terapeuta. ${ }^{4}$ Morse e colaboradores, por sua vez, definiram quatro componentes da empatia: emotivo, cognitivo, moral e comportamental. ${ }^{5}$

A capacidade de estabelecer empatia integra o eixo central da prática em medicina geral e familiar (MGF), ${ }^{4}$ baseada numa medicina centrada na pessoa e numa abordagem holística ${ }^{3}$ e baseada no estabelecimento de uma relação terapêutica. ${ }^{6}$ Como ferramenta utilizada na construção da re- lação médico-doente interfere positivamente na qualidade dos cuidados de saúde prestados e na satisfação do médico e do doente o que, por sua vez, melhora a acuidade diagnóstica e a adesão terapêutica. ${ }^{7}$ Estudos mostram que uma comunicação empática exerce uma influência positiva, não só na saúde mental, mas também na resolução dos sintomas, estados funcionais e fisiológicos e controlo da dor. ${ }^{8}$

Numa revisão sistemática de 2013 foi estudada a efetividade da empatia na prática clínica dos cuidados de saúde primários (CSP) e identificou-se uma correlação positiva entre as competências empáticas do médico e a satisfação do doente, com reflexo na diminuição dos níveis de ansiedade e melhoria dos resultados em saúde. ${ }^{9}$

Como conceito subjetivo, a sua caracterização e quantifi-

1. Médico Interno de Medicina Geral e Familiar. USF Carcavelos

2. Médica Interna de Medicina Geral e Familiar. UCSP Parede

3. Professor Auxiliar convidado. Unidade de Medicina Geral e Familiar, NOVA Medical School

4. Assistente Graduado Sénior. USF Carcavelos, ACeS Cascais 
cação são um desafio. Carl Rogers, nos seus trabalhos durante a década de 50, elaborou diversas escalas de quantificação aplicadas à psiquiatria ou a contextos de internamento, sendo colocada em questão a sua aplicabilidade nos cuidados de saúde primários e questionada a sua capacidade para refletir a visão dos doentes. ${ }^{10}$ Por este motivo, desde 2001 tem sido construída e validada uma escala de avaliação da empatia médica, denominada de Escala de Empatia Médica de Jefferson (JSPE), que se relaciona com competências clínicas e relacionais da prática médica e que já passou por um processo de validação para a população médica portuguesa ${ }^{2}$ e para estudantes de medicina. ${ }^{11}$ Esta escala pontua a perceção de empatia de forma crescente, variando entre 0 e 140. Num estudo desenvolvido durante onze anos com aplicação da escala a 2.637 estudantes de medicina procurou-se uma normalização dos resultados, definindo-se um limiar de resposta baixa em 95 (sexo masculino) e 100 pontos (sexo feminino). ${ }^{12}$

Diversos estudos apontam para diferentes graus de modificação dos níveis de empatia em estudantes de medicina durante os seis anos de curso, mas com uma diferença estatisticamente significativa consoante o género, identificando-se consistentemente maior capacidade empática por parte do sexo feminino. ${ }^{11-14} \mathrm{Um}$ estudo coorte realizado em internos de pediatria demonstrou não haver decréscimo dos níveis de empatia num período de dois anos de formação específica. ${ }^{15}$

A relação médico-doente e o estabelecimento de empatia têm ganho crescente peso na formação pré e pós-graduada e existem já alguns protocolos de ensino de técnicas relacionais baseadas na neurobiologia das emoções ${ }^{8} \mathrm{ou}$ através do treino em teatro ${ }^{16}$ que parecem apresentar um impacto positivo na empatia. O núcleo de competências que recebe maior destaque engloba a capacidade de deteção de emoções, no próprio e no doente, através de linguagem verbal e não-verbal; a capacidade de responder de forma empática; a capacitação do médico no autoconhecimento e autocontrolo emocional e psicológico; e a capacidade de aplicar estas técnicas na comunicação com o doente, sendo capaz de fornecer apoio e acompanhamento na resolução de desafios comunicacionais. ${ }^{17}$

Sendo uma ferramenta essencial para o médico de família, desconhece-se o impacto que a atual formação específica em MGF em Portugal tem nas competências empáticas dos internos.

\section{MÉTODOS}

O estudo englobou a população de internos em formação específica (FE) em MGF, definindo-se uma amostra de conveniência composta por dois grupos de internos:

- Grupo 2012: internos que iniciaram a FE no ano de 2012 (tendo completado quatro anos de internato);

- Grupo 2016: internos que iniciaram a FE em 2016, a nível nacional.

Os critérios de exclusão definidos contemplavam todos os internos não pertencentes aos grupos definidos anteriormente (grupo 2012 e 2016).

Foi calculada estatisticamente a dimensão da amostra com recurso ao programa Power/sample size calculator, do departamento de estatística da University of British Columbia (Canadá).$^{18}$ Para uma diferença esperada entre médias de 5 valores (diferença encontrada em estudos prévios) ${ }^{10-11,13}$ e um desvio-padrão aproximado de 10, utilizando um valor de erro tipo alfa de $5 \%$ e para uma potência de $80 \%$, determinou-se a necessidade de pelo menos 63 respostas válidas em cada grupo de internos.

Elaborou-se um questionário anónimo e de autopreenchimento voluntário constituído por duas partes: a primeira parte com dados de caracterização do participante (idade e sexo), local de formação pré-graduada e de formação específica e aferição do grau de experiência prévia em comunicação (formação obrigatória ou opcional em comunicação durante a formação pré-graduada, cursos/workshops frequentados de forma voluntária durante a formação pré e pós-graduada); a segunda parte constituída pela JSPE - versão traduzida e validada para Portugal.

A JSPE é composta por vinte itens quantificados segundo uma escala de Likert, de um a sete, agrupados em três fatores com valor próprio: "tomada de perspetiva» (dez items), «compaixão» (sete items) e «capacidade de se colocar no lugar do paciente» (dois items).

O protocolo e o questionário foram submetidos à Comissão de Ética, tendo obtido parecer favorável. Inicialmente foi pedida a colaboração das coordenações de internato (CI) por correio eletrónico. No e-mail enviado constava um texto introdutório com uma breve explicação do objetivo do estudo e um convite à participação voluntária e anónima, acedendo ao questionário on-line através de uma hiperligação. Nos casos em que não se obteve resposta por parte da $\mathrm{CI}$ foi solicitado apoio às respetivas comissões de internos para a divulgação do estudo.

O preenchimento do questionário foi feito on-line, exceto no caso da CI da ARS de Lisboa eVale do Tejo (ARSLVT) onde foi possível a aplicação do questionário de forma presencial e em formato de papel em reuniões com os internos, entre janeiro e março de 2016.

Durante o período de colheita de dados (janeiro a maio 


\begin{tabular}{|c|c|c|c|}
\hline & GRUPO 2012 & GRUPO 2016 & Valor $p$ \\
\hline Género do participante, n(\%) & & & 0,466 a) \\
\hline Feminino & $64(76,2)$ & $176(80,0)$ & \\
\hline Masculino & $20(23,8)$ & $44(20,0)$ & \\
\hline Idade (anos), mediana (min-máx) & $30,00(26-47)$ & $26,00(24-38)$ & $<0,001 \mathrm{~b})$ \\
\hline ARS onde realizam FE, $n(\%)$ & & & $0,344 a)^{*}$ \\
\hline Norte & $33(39,3)$ & $72(32,7)$ & \\
\hline LVT & $44(52,4)$ & $135(61,4)$ & \\
\hline Alentejo & $2(2,4)$ & $5(2,3)$ & \\
\hline Algarve & $3(3,6)$ & $3(1,4)$ & \\
\hline Madeira & $2(2,4)$ & $5(2,3)$ & \\
\hline Formação pré-graduada, n(\%) & & & $0,382 \mathrm{a})^{* *}$ \\
\hline Norte & $22(27,50)$ & $51(23,61)$ & \\
\hline Centro & $13(16,25)$ & $34(15,74)$ & \\
\hline LVT & $34(42,50)$ & $111(52,40)$ & \\
\hline Algarve & $0(0,00)$ & $4(1,85)$ & \\
\hline Estrangeiro & $12(14,80)$ & $16(7,40)$ & \\
\hline Formação anterior em comunicação, n(\%) & & & $<0,001$ a) \\
\hline Sim & $83(98,8)$ & $178(80,9)$ & \\
\hline Não & $1(1,2)$ & $42(19,1)$ & \\
\hline
\end{tabular}

Legenda:

a) Teste de Qui-quadrado; b) Teste não paramétrico de Mann-Whitney; NA - Não aplicável.

*Valor obtido aglutinando as respostas «Alentejo», «Algarve» e «Madeira», de modo a aplicar o teste.

**Valor obtido aglutinando as respostas «Algarve» e «Estrangeiro», de modo a aplicar o teste.

mação anterior $v s$. sem formação anterior) face a uma variável quantitativa (pontuação na JSPE) foi efetuada através do teste não paramétrico de Mann-Whitney, uma vez que os pressupostos para realização do teste $t$ não foram verificados. Os testes estatísticos foram efetuados considerando um nível de significância de $5 \%$.

\section{RESULTADOS}

O estudo inclui 304 questionários válidos: $84(27,7 \%)$ do grupo $2012 \mathrm{e}$ 220 (72,4\%) do grupo 2016. A taxa de resposta após o primeiro envio foi de $15,2 \%$ para o grupo 2012 e de $39,1 \%$ para o grupo 2016 , atingindo um valor final de $20,6 \%$ e $42,3 \%$, respetivamente, após novo envio do formulário. No grupo 2016, a totalidade dos questionários aplicados na ARSLVT foi obtida presencialmente.

Com a análise comparativa dos dois grupos (Quadro I) constata-se que não existem diferenças estatisticamente significativas quanto ao género ( $p=0,446)$, faculdade de formação prégraduada ( $p=0,382)$ e ARS onde realizam a FE $(p=0,344)$. A diferença en-

de 2016) realizou-se novo envio do formulário on-line para todas as ARS com o objetivo de relembrar e estimular a participação no estudo. De forma a evitar duplicação de respostas neste inquérito, a hiperligação para o formulário foi enviada para o $e$-mail pessoal de cada interno através da respetiva coordenação de internato ou comissão de internos, sendo possível apenas uma submissão de resposta por cada $e$-mail contactado.

Os resultados obtidos foram inicialmente organizados por dois dos autores numa base de dados do Microsoft Excel 2013 e posteriormente analisados estatisticamente através do software estatístico IBM® SPSS Statistics, v. 19. As variáveis quantitativas foram sumariadas através de estatísticas descritivas, nomeadamente frequência absoluta (n), mediana, mínimo e máximo, e as variáveis qualitativas através de frequência absoluta e relativa (\%). A associação entre as variáveis qualitativas foi testada através do teste do Qui-Quadrado. A comparação entre grupos independentes (grupo 2012 vs. grupo 2016; feminino vs. masculino e forcontrada em relação à idade $(p<0,001)$ justifica-se pela diferente fase de formação. Verifica-se também que em ambos os grupos a maioria dos internos pertencem à ARSLVT e que realizaram a sua formação pré-graduada em Lisboa. A proporção de indivíduos com formação anterior em comunicação é mais elevada nos internos do grupo 2012, sendo essa diferença estatisticamente significativa $(p<0,001)$.

Em relação à pontuação obtida com a aplicação da JSPE (Quadro II) constata-se que não existem diferenças estatisticamente significativas nos dois grupos, mas que ambos se encontram acima do valor de corte designado para a escala.

Identificaram-se 11 participantes abaixo do limiar de pontuação normal, seis $(7,14 \%)$ do grupo 2012 e cinco (2,27\%) do grupo 2016.

Quanto aos fatores que poderão fazer variar a pontuação da JSPE (Quadro III) verificou-se que a experiência prévia na área da comunicação parece não se relacionar com maior pontuação, ainda que possam haver variáveis de confundimento não estudadas. Em relação ao género 


$\begin{aligned} & \text { QUADRO II. Análise comparativa dos resultados obtidos com a } \\
& \text { aplicação da JSPE em ambos os grupos }\end{aligned}$
\begin{tabular}{l|c|c|c} 
Pontuação na JSPE & GRUPO 2012 & GRUPO 2016 & Valor $\boldsymbol{P}$ \\
\hline Mediana (min-máx) & 119,00 (79-133) & $118,00(95-137)$ & 0,678 a)
\end{tabular}

Legenda: a) Teste não paramétrico de Mann-Whitney.

\begin{tabular}{|c|c|c|}
\hline & Mediana (min-max) & Valor $p$ \\
\hline Género, mediana (min-máx) & & 0,008 a) \\
\hline Feminino & $119,00(74-140)$ & \\
\hline Masculino & $115,00(80-137)$ & \\
\hline Formação anterior, mediana (min-máx) & & 0,610 a) \\
\hline Sim & $116,00(99-135)$ & \\
\hline Não & $118,00(74-140)$ & \\
\hline
\end{tabular}

Legenda: a) Teste não paramétrico de Mann-Whitney.

feminino apresenta um nível de empatia estatisticamente superior ao do género masculino $(p=0,008)$.

\section{DISCUSSÃO}

No presente estudo, os autores pretenderam avaliar o impacto da FE em MGF nas competências empáticas dos internos em Portugal. No que se refere ao nível de empatia quantificado pela escala de Jefferson, traduzida e validada para a população médica portuguesa, o valor foi semelhante $(p=0,678)$ entre os dois grupos analisados (internos que iniciaram a sua FE em 2012 e em 2016). Os valores obtidos são concordantes com diversos estudos realizados em alunos em formação pré-graduada e internos de outras especialidades, ${ }^{12,15}$ em que não se identificou variação do nível de empatia ao longo dos anos de formação.

Neste estudo obtiveram-se valores médios e medianos de empatia semelhantes a estudos nacionais e internacionais, ${ }^{11-14}$ tendo-se verificado um nível superior de empatia nos elementos do sexo feminino $(p=0,008)$, em linha com a literatura existente. ${ }^{11-14}$ Os grupos estudados são comparáveis entre si nas variáveis analisadas (género, faculdade de formação pré-graduada e ARS de FE). Os resultados obtidos podem representar valores elevados de empatia a priori, já que os internos que escolhem especialidades "orientadas para o paciente" pontuam mais alto do que aqueles que escolhem especialidades "orientadas para tecnologia”. Alguns estudos apontam um efeito negativo da fase clínica da formação médica nos níveis de empatia em oposição a um desenvolvimento de competências técnicas. Neste sentido, os resultados podem ser interpretados positivamente pela semelhança no nível de empatia entre os dois grupos.

A amostra selecionada apresenta uma elevada percentagem de internos da ARSLVT, não tendo sido possível obter respostas da ARS do Centro e Açores, condicionando um viés de amostragem importante. A utilização de uma amostra de conveniência de internos nos extremos da sua FE pode ter condicionado um viés de amostragem, mas deveu-se à tentativa de se encontrar uma diferença mensurável. Apesar do envio múltiplo de formulários através de correio eletrónico, a baixa taxa de resposta limita a representatividade da amostra. A metodologia utilizada neste estudo, com a utilização de uma escala validada e de aplicação transversal em diversos estudos, permite a comparação dos dados obtidos com a literatura nacional e internacional. Por outro lado, persiste a discussão sobre a aplicabilidade de escalas quantitativas de autopreenchimento na avaliação de competências subjetivas, ainda que o estudo original e os estudos de validação tenham demonstrado que pontuações mais elevadas na JSPE estão diretamente associadas a um melhor aproveitamento nas competências clínicas e práticas e, consequentemente, na satisfação dos doentes.

Novos estudos são necessários no sentido de confirmar a tendência identificada nos resultados obtidos, nomeadamente através da utilização de diferentes metodologias como o seguimento de coortes de internos. A comparação com internatos de diferentes especialidades poderá igualmente ajudar na avaliação do atual currículo da especialidade de MGF. Apesar das limitações discutidas, a utilização de escalas validadas como a JSPE parece apresentar-se como uma estratégia padronizada e objetiva de avaliação das competências humanas e relacionais essenciais ao estabelecimento de empatia. Esta ferramenta poderá ser útil na avaliação de estratégias formativas a aplicar no desenvolvimento de competências comunicacionais durante o internato.

\section{AGRADECIMENTOS}

Os autores agradecem à Coordenação de Internato da ARS LVT e em particular à Prof. ${ }^{a}$ Dr. ${ }^{a}$ Isabel Santos e ao Dr. David Rodrigues, pela revisão do protocolo e apoio na divulgação. Agradecem igualmente ao departamento de MGF da NOVA Medical School pelos importantes contributos na melhoria do trabalho e à CLINICAL $L A B$, em particular à Dra. Filipa Negreiro, pelo contributo na análise estatística e a todos os internos participantes. 


\section{REFERÊNCIAS BIBLIOGRÁFICAS}

1. Nunes JM. Comunicação em contexto clínico [Internet]. Lisboa: Bayer Health; 2007. Available from: http://www.mgfamiliar.net/itemgenerico/comunicacaoem-contexto-clinico

2. Aguiar P, Salgueira A, Frada T, Costa MJ. Empatia médica: tradução, validação e aplicação de um instrumento de medição. In: Actas do X Congresso Internacional Galego-Português de Psicopedagogia [Internet]. Braga: Universidade do Minho; 2009. p. 3705-16. Available from: http://www.educacion.udc.es/grupos/gipdae/documentos/congreso/Xcongreso/pdfs/t8/t8c272.pdf

3. Macedo A, Cavadas LF, Sousa M, Pires P, Santos JA, Macha A. Empathy in family medicine. Rev Port Clin Geral. 2011;27(6):527-32.

4. Barrett-Lennard GT. The empathy cycle: refinement of a nuclear concept. J Counsel Psychol. 1981;28(2):91-100.

5. Aomatsu M, Otani T, Tanaka A, Ban N, van Dalen J. Medical students' and residents' conceptual structure of empathy: a qualitative study. Educ Health (Abingdon). 2013;26(1):4-8.

6. McWhinney IR, Freeman T. Textbook of family medicine. 3rd ed. New York: Oxford University Press; 2009. ISBN 9780195369854

7. Mercer SW, Reynolds WJ. Empathy and quality of care. Br J Gen Pract. 2002;52(Suppl):S9-12.

8. Riess H, Kelley JM, Bailey RW, Dunn EJ, Phillips M. Empathy training for resident physicians: a randomized controlled trial of a neuroscience-informed curriculum. J Gen Intern Med. 2012;27(10):1280-6.

9. Derksen F, Bensing J, Lagro-Janssen A. Effectiveness of empathy in general practice: a systematic review. Br J Gen Pract. 2013;63(606):e76-84.

10. ReynoldsWJ.The measurement and development of empathy in nursing.Aldershot: Ashgate; 2000. ISBN 9780754612643

11. Loureiro J, Gonçalves-Pereira M, Trancas B, Caldas-de-Almeida JM, Castro-Caldas A. Empatia na relação médico-doente: evolução em alunos do primeiro ano de medicina e contribuição para a validação da Escala Jefferson em Portugal [Empathy in the doctor-patient relationship as viewed by first-year medical stu- dents: data on validity and sensibility to change of the Jefferson Measure in Portugal]. Acta Med Port. 2011;24(S2):431-42. Portuguese

12. Hojat M, Gonnella JS. Eleven years of data on the Jefferson Scale of Empathy Medical Student Version (JSE-S): proxy norm data and tentative cut-off scores. Med Princ Pract. 2015;24(4):344-50.

13. Magalhães E, Salgueira AP, Costa $P$, Costa MJ. Empathy in senior year and first year medical students: a cross-sectional study. BMC Med Educ. 2011;11:52.

14. Quince TA, Parker RA, Wood DF, Benson JA. Stability of empathy among undergraduate medical students: a longitudinal study at one UK medical school. BMC Med Educ. 2011;11:90

15. Greenberg L, Agrawal D, Toto R, Blatt B. Empathy, sense of power and personality: do they change during pediatric residency? South Med J. 2015;108(8):471-4.

16. Dow AW, Leong D, Anderson A, Wenzel RP. Using theater to teach clinical empathy: a pilot study. J Gen Intern Med. 2007;22(8):1114-8.

17. Stein T, Frankel RM, Krupat E. Enhancing clinical communication skills in a large healthcare organization: a longitudinal case study. Patient Educ Couns. 2005;58(1):4-12.

18. Brant R. Power/Sample size calculator - Inference for means: comparing two independent samples [Internet]. Vancouver: University of British Columbia; [cited 2016 Dec 20]. Available from: https://www.stat.ubc.ca/ rollin/stats /ssize/n2.html

\section{CONFLITO DE INTERESSES}

Os autores declaram não ter conflitos de interesses.

\section{ENDEREÇO PARA CORRESPONDÊNCIA}

Nuno Basílio

E-mail: nunomdbasilio@gmail.com

Recebido em 26-10-2016

Aceite para publicação em 30-05-2017

\section{ABSTRACT}

\section{EMPATHY IN FAMILY MEDICINE TRAINEES}

Aim: The aim of this study was to assess empathy in family medicine trainees and to compare the level of empathy in first and final year trainees.

Type of study: Cross-sectional.

Location: Healthcare Regional Administrations (HRA) in Portugal.

Population: Family medicine trainees in Portuguese HRA.

Methods: We gave an anonymous, self-administered questionnaire, including the Jefferson Scale of Physician Empathy (JSPE), adapted and validated for Portugal, to a convenience sample of family medicine residents. The surveys were distributed in person or sent by personal e-mail. Statistical analysis was performed using SPSS (R) 19.0.

Results: We collected 304 questionnaires. Of these, 84 were from trainees who began their training in 2012 (response rate (RR) $=20.6 \%$ ). The remaining 220 questionnaires were from trainees who began their training in 2016 ( $R R=42.3 \%)$. Most of the trainees were enrolled in programs in the Lisbon and Tejo Valley regions (52.4\% and 61.4\% respectively). There were no significant differences in gender between the two groups. There were no significant differences in the median levels $(M)$ of empathy $[M E(2012)=119.00$; $\operatorname{ME}(2016)=118.00 ; p=0.678$ ] between the first and final year trainees. Prior training in communication skills was not associated with a higher empathy score. Female participants had significantly higher empathy scores $(p=0.008)$.

Conclusions: Our results were comparable to national and international studies on empathy in medical students and trainees. Empathy does not seem to be associated with residency or undergraduate training. The low response rate obtained limits the representativeness of the sample and the validity of the findings. However this study presents an initial look at the variation in empathy during family medicine residency training in Portugal.

Keywords: Empathy; Residency; General practice and family medicine; Primary care. 